\title{
Modifications of the Beam Profile in the New Inclined Crystal Geometry*
}

ANL/CP- -74077

DE 92003446

\author{
W.K. Lee and A.T. Macrander \\ Advanced Photon Source \\ Argonne National Laboratory \\ 9700 South Cass Avenue \\ Argonne, IL 60439
}

October, 1991

$\mathrm{cz}$

\begin{abstract}
*This work supported by the U.S. Department of Energy, BES-Materials Sciences, under contract no. W-31-109-ENG-38
\end{abstract}

\section{DISCLAIMER}

This report was prepared as an account of work sponsored by an agency of the United States Government. Neither the United States Government nor any agency thereof, nor any of their employees, makes any warranty, express or implied, or assumes any legal liability or responsibility for the accuracy, completeness, or usefulness of any information, apparatus, product, or process disclosed, or represents that its use would not infringe privately owned rights. Reference herein to any specific commercial product, process, or service by trade name, trademark, manufacturer, or otherwise does not necessarily constitute or imply its endorsement, recommendation, or favoring by the United States Government or any agency thereof. The views and opinions of authors expressed herein do not necessarily state or reflect those of the United States Government or any agency thereof. 


\title{
MODIFICATIONS OF THE BEAM PROFILE IN THE NEW INCLINED CRYSTAL GEOMETRY
}

\author{
Wah Keat Lee and Albert T. Macrander \\ Advanced Photon Source \\ Argonne National Laboratory \\ 9700 S. Cass Avenue \\ Argonne, IL 60439 \\ U.S.A.
}

\begin{abstract}
The new inclined crystal geometry has been successfully used in high heat load X-ray monochromator tests. The important aspect of this geometry is that from a diffraction point of view, when properly aligned, it is a symmetric Bragg reflection; i.e., $b=k_{i} \cdot n / k_{\text {out }} \cdot \mathbf{n}=-1$. An interesting result of this geometry is that with a single reflection from an inclined crystal, the output $X$-ray beam shape changes dramatically, while maintaining the same beam cross sectional area. For example, a parallel $8 \mathrm{keV}$ input $X$-ray beam using $\mathrm{Si}(111)$ reflection, with an inclination angle of $70.5^{\circ}$, the output beam size is compressed by about a factor of 5.4 in one direction and expanded by the same factor in the other direction. This geometry can therefore, be used to alter the source line profile of in house $X$-ray generators and in some cases, be used to better match the sample size and the $X$-ray beam. The effect of this geometry on beam profiles, beam divergences and acceptance angles will be discussed.
\end{abstract}


One of the most challenging problems confronting beam line designers for the third generation synchrotron radiation sources such as the Advanced Photon Source (APS), the European Synchrotron Radiation Facility (ESRF) and the Super Photon Ring (SPring-8), is the high power and power densities delivered to the first optical elements. At $30 \mathrm{~m}$ from the source, the APS $2.5 \mathrm{~m}$ wiggler A delivers about $2.3 \mathrm{~kW}$ of power over $142 \mathrm{~mm}^{2}$, while the APS $2.5 \mathrm{~m}$ undulator A delivers $5 \mathrm{~kW}$ of total power, with peak power densities of $180 \mathrm{~W} / \mathrm{mm}^{2}$. At such high power and in particular, high power density, the thermally induced strain on an uncooled silicon crystal monochromator becomes intolerable. Various cooling methods and geometries are currently being pursued by different groups. One of the interesting directions in this effort is the so called "inclined crystal geometry", developed at the APS [1,2].

The inclined crystal geometry monochromator has been successfully tested at the CHESS undulator at power densities of $40 \mathrm{~W} / \mathrm{mm}^{2}$ without any observable thermally induced crystal strain [3]. The primary advantage of the inclined crystal geometry is the enlargement of the beam footprint on the face of the crystal, thus, decreasing the power density as seen by the crystal. This is particularly important in the case of undulator or focussed beams. Although the idea of spreading out the beam footprint is not new [4], the idea that one can spread out the beam footprint while maintaining a symmetric Bragg reflection is new. This is the crucial point of this inclined geometry. When the crystal is properly aligned, it is $a b=$ -1 symmetric Bragg reflection; where $b$, the asymmetry factor is defined as $\mathbf{k}_{\mathbf{i}} \cdot \mathbf{n} / \mathbf{k}_{\text {out }} \cdot \mathbf{n}$, and $\mathbf{k}_{\mathbf{i n}}, \mathbf{k}_{\text {out }}$, and $\mathbf{n}$ are the incoming wavevector, outgoing wavevector and the crystal surface normal respectively. The alignment aspects of this geometry is described in another paper [5]. An interesting result of this geometry is that with a single reflection from an inclined crystal, the outgoing beam profile is no longer the same as that of the incoming beam. When required, a second similarly inclined crystal can be used to recover the original beam profile. This paper describes some aspects of this beam profile modification and suggests some possible interesting uses.

Figure 1 shows the inclined crystal geometry for a parallel incoming beam with a square cross sectional area. $\theta$ is defined as the between the $X$-ray beam and the Bragg planes, and $\beta$ is defined as the angle between the surface and the Bragg planes and is often refered to as the inclination angle. In the normal flat geometry, $\beta=0$. As can be seen, in the inclined 
geometry, although the normal to the surface, $n$, does not coincide with the reciprocal lattice vector, $\mathbf{H}$, it lies in a plane that is normal to the scattering plane as defined by the incoming and outgoing wave vectors. Therefore, $\mathbf{k}_{\mathbf{i}} \cdot \mathbf{n}=-\mathbf{k}_{\text {out }} \cdot \mathbf{n}$ and thus, $b=-1$. The Darwin widths and acceptance of such a reflection is the same as that of a symmetric Bragg reflection. The beam profiles and beam footprint on the surface of the crystal for both the inclined and the conventional flat geometries are shown in figure 2. As can be seen, the outgoing beam profile of the inclined geometry is dramatically different than that of the incoming beam. However, it is important to note that the beam cross sectional area remains the same. Also, since the angular properties of the beam remain unchanged $(b=-1)$, the well known Louiville's phase space conservation theorem is not violated. The area of the beam footprint on the surface of the crystal is however, greatly increased. With an inclination of $70.5^{\circ}$, the beam footprint area is 3 times that of the normal flat geometry. The outgoing beam profile is extended 5.4 times in one direction while compressed by the same amount in the other direction. In general, the amount of extension (the length of the long edge in figure $2(e)$ ) is given by:

$$
\left[4 \cos ^{2} \theta \tan ^{2} \beta+1\right]^{1 / 2}
$$

The outgoing beam remains parallel because it is a symmetric reflection.

We now consider the case for an angularly divergent incoming beam. For ease of illustration, figure 3 shows the case of a vertically divergent beam that has negligible horizontal size and figure 4 shows the case of a horizontally divergent beam with negligible vertical size. In both figures, the beam footprints on the crystal surface are the bold lines $A C$, and it is assumed that the central beams $O B$ and $B E$ satisfy the symmetric Bragg reflection requirement that $b=-1$. In both the flat and inclined cases, the vertical (as defined in figure 3 ) divergence of the incoming beam merely changes the value of $\theta$. The asymmetry parameter $b$ remains -1 . For an incoming beam with horizontal divergence, in the flat geometry case, since the surface normal $\mathrm{n}$ is parallel to the reciprocal lattice vector $\mathrm{H}$, and $\mathbf{k}_{\mathbf{j}} \cdot \mathbf{H}=-\mathbf{k}_{\text {out }} \cdot \mathbf{H}$, the reflection remains symmetric for all the rays. However, this is not true for the inclined case since the surface normal is no longer parallel to $\mathrm{H}$. Referring to figure 4 , we define a positive divergent angle as one that results in a more grazing incidence angle (ray $\mathrm{OC}$ ) and a negative divergent angle as one that results in a less grazing incidence angle (ray $O A$ ), with the angles measured with respect to the 
central beam $O B$. Equation (2) describes the relationship between an incoming ray at some divergent angle $\delta$ (as measured from the central ray). Here, $\theta$ is defined as the angle that the central beam OB makes with the Bragg planes. Notice that there is a different asymmetric parameter $b$ for each different ray of the incoming beam. Figure 5 is a plot of equation (2) for an $8 \mathrm{keV} \mathrm{Si}(111)$ reflection $\left(\theta=14.22^{\circ}\right)$ with an inclination angle of $70.5^{\circ}$.

$$
b=\frac{\sin \delta \sin \beta-\sin \theta \cos \beta \cos \delta}{\sin \delta \sin \beta+\sin \theta \cos \beta \cos \delta}
$$

The typical bending magnet beam line at the APS can accept about \pm 3 mrad $\left( \pm 0.2^{\circ}\right)$ of radiation in the orbital (horizontal) plane. For $8 \mathrm{keV}, \mathrm{Si}$ (111) reflection, the horizontal beam divergence in this case will cause $b$ to vary between -0.92 and -1.08 . Since the input acceptance angle varies as $|b|^{-1 / 2}$, and the output divergence varies as $|b|^{1 / 2}$, the change in the angular properties of the beam due to the divergence of the incoming beam is less than $4 \%$. Therefore, the divergence of the outgoing beam remains essentially the same as that of the incoming beam. Thus, we see that although a reflection in this inclined geometry with a divergent beam may cause some asymmetry in the output beam properties, the effect is usually small.

Finally, we would like to discuss an interesting possible use for this inclined geometry. Although we described above the case of a small square incoming beam and an elongated outgoing beam, the vice versa case is obviously also possible; that is, with an elongated input beam and a squarish outgoing beam. Thus, we have a way of modifying the beam cross sectional profiles without affecting the beam angular properties. One possible use of this is to match the shape of the incoming radiation profile to a particular sample shape, and thus increasing the number of photons hitting the sample. This can be particularly helpful in the case of synchrotron bending magnet sources where the radiation is usually quite extended in the horizontal orbital plane and narrow in the vertical plane. If the sample is more or less circular in cross section, such that the diameter is larger than the vertical extent of the beam, but smaller than the horizontal extent, then, by properly aligning the inclined 
monochromator, it is possible to divert more photons onto the sample. This technique can also be used to modify the beam profiles from in-house $\mathrm{X}$-ray generators.

In summary, the new inclined crystal geometry provides us with a way to modify the shape of $X$-ray beam profiles while maintaining $a b=-1$ symmetric Bragg reflection. It is important to remember however, that this is not a true foclissing geometry. Although the shape of the beam profile is changed, the cross sectional area of the beam remains the same. In other words, the brilliance (number of photons $/ \mathrm{s} .0 .1 \% \mathrm{BW} \mathrm{mrad}^{2} . \mathrm{mm}^{2}$ ) is unchanged.

This work is supported by the U. S. Department of Energy, BES-Materials Science, under Contract No. W-31-109-ENG-38.

References:

[1] A. Khounsary, A. Macrander, W. K. Lee, D. Mills, R. Smither, and W. Yun, Workshop on Thermal Problems of Synchrotron Radiation Optics, held as a satellite meeting of the International Synchrotron Radiation Instrumentation meeting, July 20, 1991, Chester College, U. K.

[2] W. K. Lee, A. Macrander, D. Mills, R. Smither, and A. Khounsary, Workshop on Thermal Problems of Synchrotron Radiation Optics, held as a satellite meeting of the International Synchrotron Radiation Instrumentation meeting, July 20, 1991, Chester College, U. K.

[3] A. T. Mlacrander, W. K. Lee, D. M. Mills, C. S. Rogers, and R. K. Smither, this proceeding.

[4] R. K. Smither, G. A> Forster, C. A. Kot, and T. M. Kuzay, Nuclear Instruments and Methods, A266, 517, (1988).

[5] A. Macrander and W. K. Lee, this proceeding. 
Figure Captions:

Figure 1:

The inclined crystal geometry for a parallel square cross sectional incoming beam. The beam footprint on the surface of the crystal is shaded black. Notice that the normal to the surface, $n$, is not parallel to the reciprocal lattice vector, $H$.

Figure 2:

Beam profiles and footprints for the flat and inclined geometries.for a square incoming beam profile. (a) Shape of incoming beam profile (b) Footprint on a normal flat crystal (c) Shape of outgoing beam profile after diffracting from a flat crystal (d) Footprint on an inclined crystal (e) Shape of outgoing beam profile after diffracting from an inclined crystal. Although the general dimensions are given, the above have been drawn to scale using $\theta=14.22^{\circ}$ and $\beta=70.5^{\circ}$.

Figure 3:

A vertically divergent beam with negligible horizoniti size impinging on an inclined crystal. The rays $O A, O B$ and $O C$ all hit the crystal at different values of $\theta$. But $b=(O A \cdot n / A D \cdot n)=(O B \cdot n / B E \cdot n)=(O C \cdot n / C F \cdot n)=-1$. Therefore this is still a symmetric Bragg reflection.

Figure 4:

A horizontally divergent beam with negligible vertical size impinging on an inclined crystal. The crystal is aligned such that the central rays $O B$ and $B E$ undergo $a b=-1$ symmetric Bragg reflection. OA hits the crystal at a less grazing incidence angle while $O C$ hits the crystal at a more grazing incidence angle. The divergent angle $\delta$ is defined as positive for the ray $O C$ and negative for the ray $O A$, measured with respect to the central ray $O B$.

Figure 5:

Plot of $b$ versus the divergent angle $\delta$ for the case of $\theta=14.22^{\circ}$ and $\beta=$ $70.5^{\circ}$. 


$$
\vartheta
$$




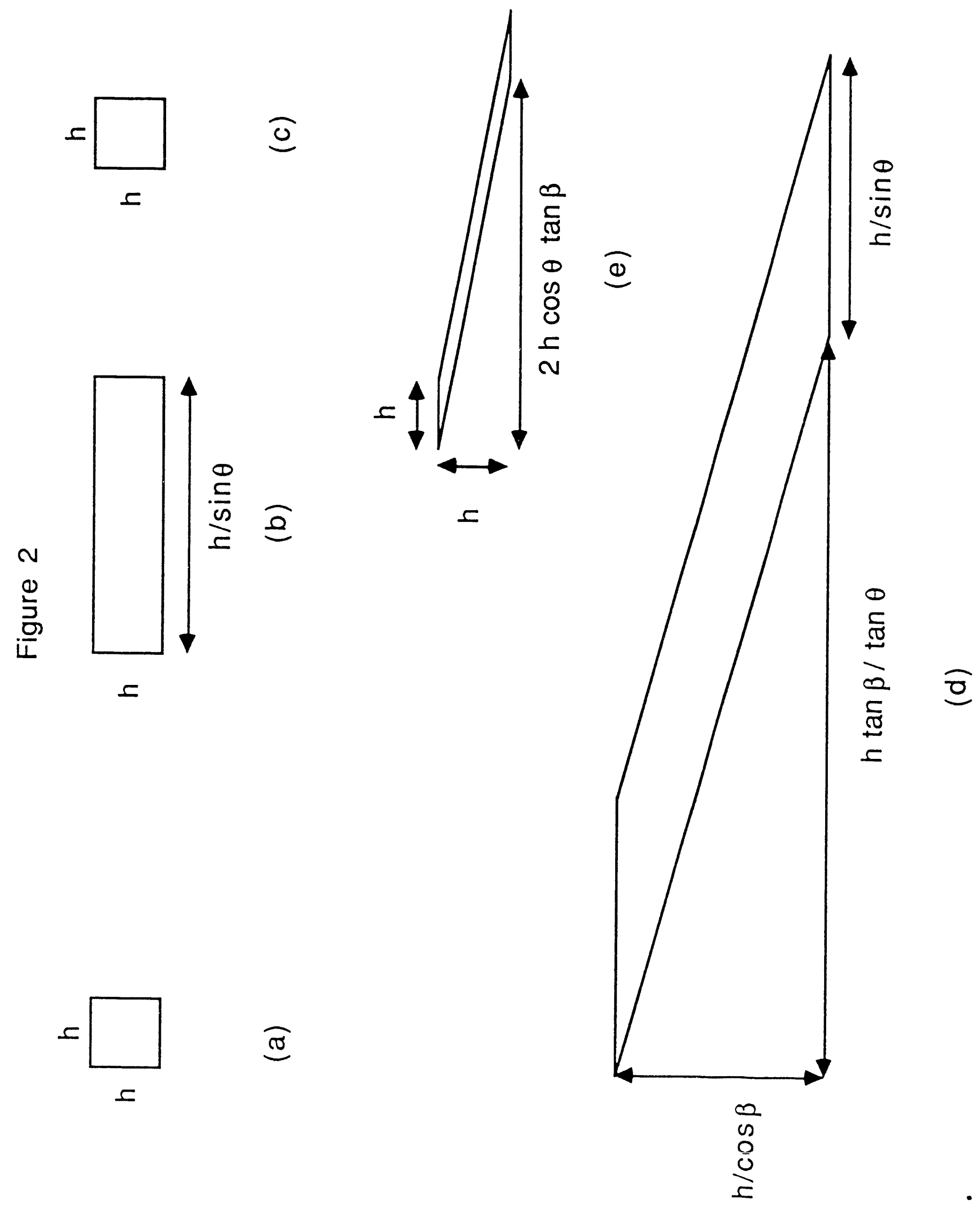




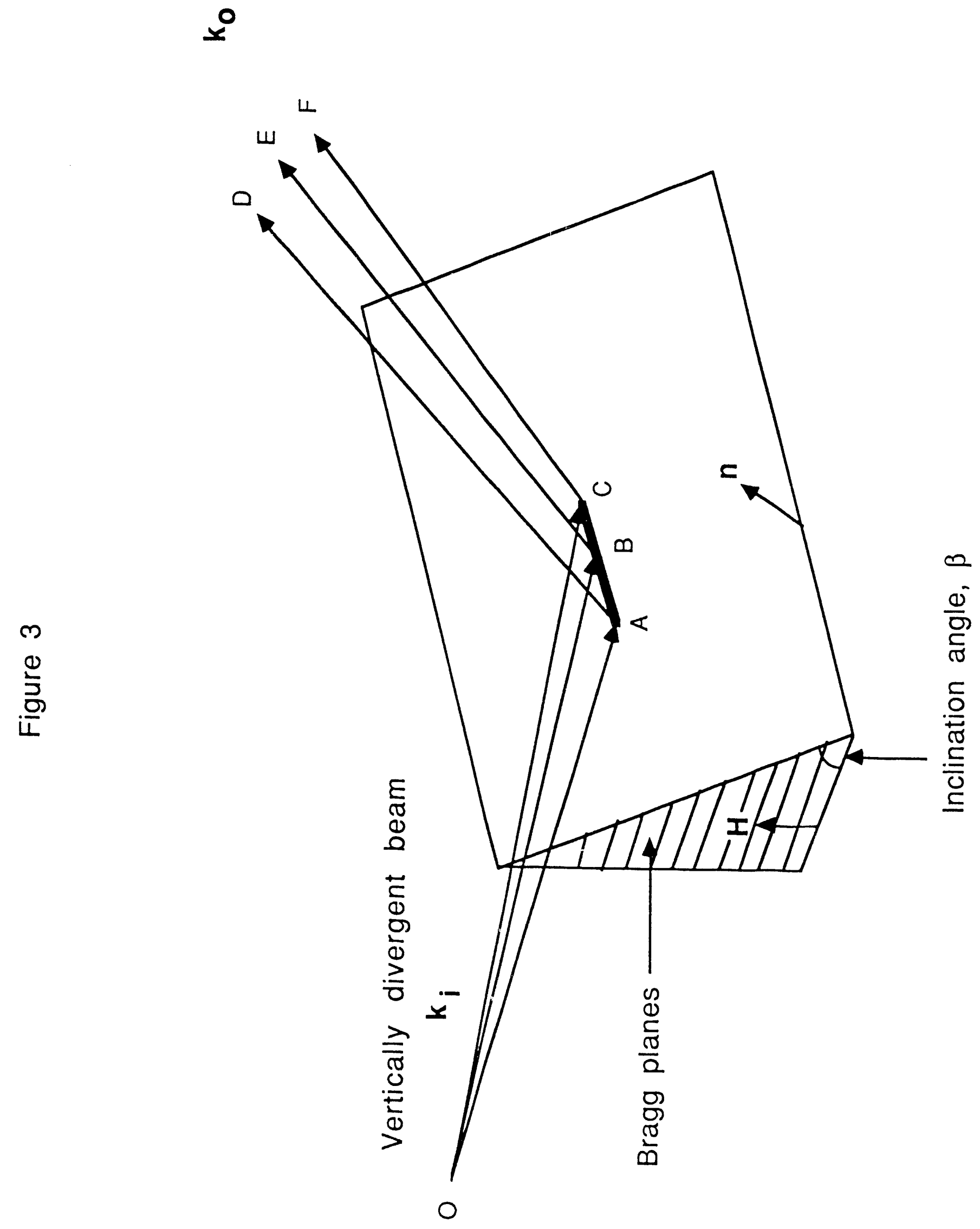




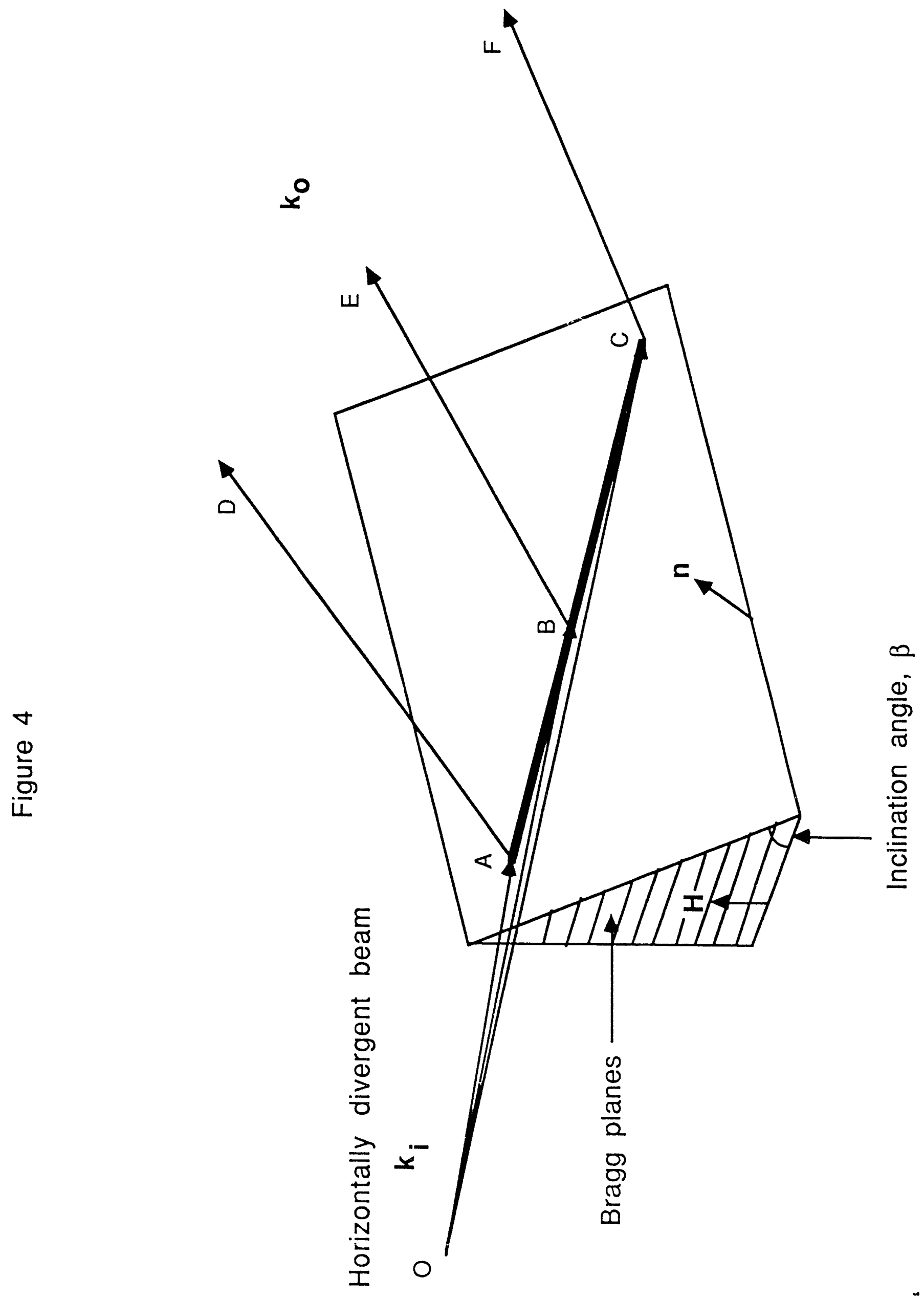




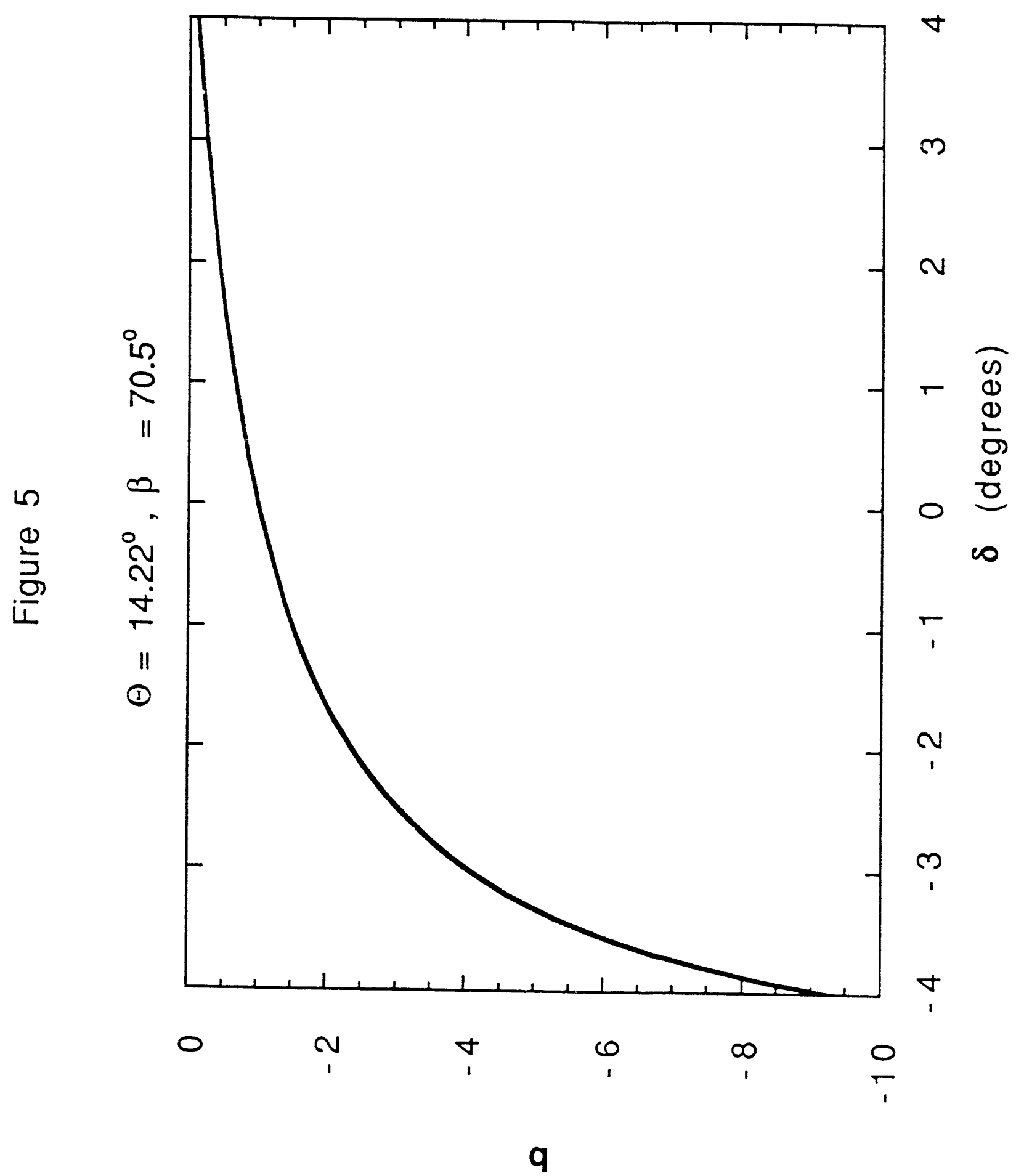





$T$ 
